\title{
Triangle-Free Triangulations, Hyperplane Arrangements and Shifted Tableaux
}

\author{
Ron M. Adin* \\ Department of Mathematics \\ Bar-Ilan University \\ Ramat-Gan 52900, Israel \\ radin@math.biu.ac.il
}

\author{
Yuval Roichman \\ Department of Mathematics \\ Bar-Ilan University \\ Ramat-Gan 52900, Israel \\ yuvalr@math.biu.ac.il
}

Submitted: Jan 18, 2012; Accepted: Aug 11, 2012; Published: Sep 6, 2012.

Mathematics Subject Classification: 05A19, 05E18, 52C35.

\begin{abstract}
Flips of diagonals in colored triangle-free triangulations of a convex polygon are interpreted as moves between two adjacent chambers in a certain graphic hyperplane arrangement. Properties of geodesics in the associated flip graph are deduced. In particular, it is shown that: (1) every diagonal is flipped exactly once in a geodesic between distinguished pairs of antipodes; (2) the number of geodesics between these antipodes is equal to twice the number of standard Young tableaux of a truncated shifted staircase shape.
\end{abstract}

\section{Introduction}

It was shown in [1] that the diameter of the flip graph on the set of all colored triangle-free triangulations of a convex $n$-gon (to be defined in Subsection 2.1) is exactly $n(n-3) / 2$. Observing that this is the number of diagonals in a convex $n$-gon, it was conjectured by Richard Stanley that all diagonals are flipped in a geodesic between two antipodes.

In this paper Stanley's conjecture is proved for distinguished pairs of antipodes (Corollary 5.3 below). The proof applies a $\widetilde{C}_{n}$-action on arc permutations, which yields an embedding of the flip graph in a graphic hyperplane arrangement. Geodesics between distinguished antipodes in the flip graph are then interpreted as minimal galleries from a given chamber $c$ to the negative chamber $-c$, while diagonals are interpreted as separating hyperplanes.

\footnotetext{
${ }^{*}$ Research of both authors was supported in part by an internal grant from Bar-Ilan University.
} 
The set of geodesics between these antipodes is further studied in Section 9. It is shown that the number of these geodesics is equal to twice the number of Young tableaux of a truncated shifted staircase shape. Motivated by this result, product formulas for this number, as well as for other truncated shapes, were found by Greta Panova [9] and Ronald C. King and the authors [2].

\section{Triangle-Free Triangulations}

In this Section we recall basic concepts and main results from [1].

\subsection{Basic Concepts}

Label the vertices of a convex $n$-gon $P_{n}(n>4)$ by the elements $0, \ldots, n-1$ of the additive cyclic group $\mathbb{Z}_{n}$. Consider a triangulation (with no extra vertices) of the polygon. Each edge of the polygon is called an external edge of the triangulation; all other edges of the triangulation are called internal edges, or chords.

Definition 2.1 A triangulation of a convex $n$-gon $P_{n}$ is called internal-triangle-free, or simply triangle-free, if it contains no triangle with 3 internal edges. The set of all trianglefree triangulations of $P_{n}$ is denoted TFT $(n)$.

A chord in $P_{n}$ is called short if it connects the vertices labeled $i-1$ and $i+1$, for some $i \in \mathbb{Z}_{n}$. A triangulation is triangle-free if and only if it contains only two short chords $[1$, Claim 2.3].

A proper coloring (or orientation) of a triangulation $T \in T F T(n)$ is a labeling of the chords by $0, \ldots, n-4$ such that

1. One of the short chords is labeled 0 .

2. If a triangle has exactly two internal edges then their labels are consective integers $i, i+1$.

It is easy to see that each $T \in T F T(n)$ has exactly two proper colorings. The set of all properly colored triangle-free triangulations is denoted $C T F T(n)$.

Each chord in a triangulation is a diagonal of a unique quadrangle (the union of two adjacent triangles). Replacing this chord by the other diagonal of that quadrangle is a flip of the chord. A flip in a colored triangulation preserves the color of the flipped diagonal.

Definition 2.2 The colored flip graph $\Gamma_{n}$ is defined as follows: the vertices are all the colored triangle-free triangulations in $C T F T(n)$. Two triangulations are connected in $\Gamma_{n}$ by an edge labeled $i$ if one is obtained from the other by a flip of the chord labeled $i$.

See Figure 2.1 for a drawing of $\Gamma_{7}$, where the coloring of a triangulation is displayed by shading the triangle with the short chord labeled 0 and two external edges as sides. 


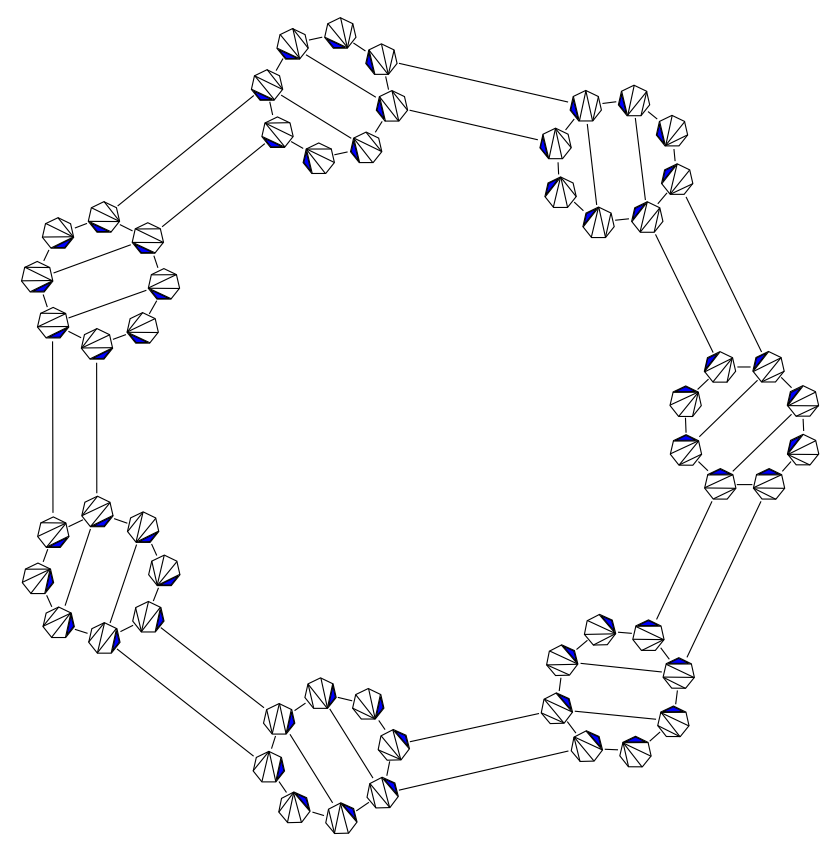

Figure 1: $\Gamma_{7}$

\subsection{A $\widetilde{C}_{n-4}$-Action on Triangle-Free Triangulations}

Let $\widetilde{C}_{n}$ be the affine Weyl group generated by

$$
S=\left\{s_{0}, s_{1}, \ldots, s_{n-1}, s_{n}\right\}
$$

subject to the Coxeter relations

$$
\begin{array}{cc}
s_{i}^{2}=1 & (\forall i), \\
\left(s_{i} s_{j}\right)^{2}=1 & (|j-i|>1), \\
\left(s_{i} s_{i+1}\right)^{3}=1 & (1 \leqslant i \leqslant n-2),
\end{array}
$$

and

$$
\left(s_{i} s_{i+1}\right)^{4}=1 \quad(i=0, n-1) .
$$

The group $\widetilde{C}_{n-4}$ acts naturally on $C T F T(n)$ by flips: Each generator $s_{i}$ flips the chord labeled $i$ in $T \in C T F T(n)$, provided that the result still belongs to $C T F T(n)$. If this is not the case then $T$ is unchanged by $s_{i}$.

Proposition 2.3 [1, Proposition 3.2] This operation determines a transitive $\widetilde{C}_{n-4}$-action on $\operatorname{CTFT}(n)$.

This affine Weyl group action on $C T F T(n)$ was used to calculate the diameter of $\Gamma_{n}$. Theorem 2.4 [1, Theorem 5.1] The diameter of $\Gamma_{n}(n>4)$ is $n(n-3) / 2$. 
For any colored triangle-free triangulation $T$, denote by $T^{R}$ the colored triangle-free triangulation obtained by reversing the labeling in $T$; namely, the chord labeled $i$ in $T$ is labeled $n-4-i$ in $T^{R}(0 \leqslant i \leqslant n-4)$.

Theorem 2.5 [1, Proposition 5.6] For every $n>4$ and $T \in C T F T(n)$, the distance between $T$ and $T^{R}$ in $\Gamma_{n}$ is exactly $n(n-3) / 2$.

\section{A $\widetilde{C}_{n-2}$-Action on Arc Permutations}

\subsection{Arc Permutations}

Let $S_{n}$ be the symmetric group on the letters $1, \ldots, n$. Denote a permutation $\pi \in S_{n}$ by the sequence $[\pi(1), \ldots, \pi(n)]$ and transpositions by $(i, j)$.

Intervals in the cyclic group $\mathbb{Z}_{n}$ are subsets of the form $\{i, i+1, \ldots, i+k\}$, where addition is modulo $n$.

Definition 3.1 A permutation $\pi \in S_{n}$ is an arc permutation if, for every $1 \leqslant k \leqslant n$, the first $k$ letters in $\pi$ form an interval in $\mathbb{Z}_{n}$ (where $n \equiv 0$, namely, the letter $n$ is identified with zero).

Example. $\pi=[1,2,5,4,3]$ is an arc permutation in $S_{5}$, but $\pi=[1,2,5,4,3,6]$ is not an arc permutation in $S_{6}$, since $\{1,2,5\}$ is an interval in $\mathbb{Z}_{5}$ but not in $\mathbb{Z}_{6}$.

The following claim is obvious.

Claim 3.2 The number of arc permutations in $S_{n}$ is $n 2^{n-2}$.

Proof. There are $n$ options for $\pi(1)$ and two options for every other letter except the last one.

Denote by $U_{n}$ the set of arc permutations in $S_{n}$.

Definition 3.3 Define $\phi: U_{n} \rightarrow \mathbb{Z}_{n} \times \mathbb{Z}_{2}^{n-2}$ as follows:

1 .

$$
\phi(\pi)_{1}:=\pi(1)
$$

2. For every $2 \leqslant i \leqslant n-1$, if $\{\pi(1), \ldots, \pi(i-1)\}$ is the arc $[k, m]$ then $\pi(i)$ is either $k-1$ or $m+1$. Let

$$
\phi(\pi)_{i}:= \begin{cases}0, & \text { if } \pi(i)=k-1 \\ 1, & \text { if } \pi(i)=m+1\end{cases}
$$

$\phi$ is clearly a bijection. 


\subsection{A $\widetilde{C}_{n-2}$-Action}

Let $\left\{\sigma_{i}: 1 \leqslant i \leqslant n-1\right\}$ be the Coxeter generating set of the symmetric group $S_{n}$, where $\sigma_{i}$ is identified with the adjacent transposition $(i, i+1)$.

Definition 3.4 For every $0 \leqslant i \leqslant n-2$ define a map $\rho_{i}: U_{n} \rightarrow U_{n}$ as follows:

$$
\rho_{i}(\pi)=\left\{\begin{array}{ll}
\pi \sigma_{i+1}, & \text { if } \pi \sigma_{i+1} \in U_{n} ; \\
\pi, & \text { otherwise. }
\end{array} \quad\left(\forall \pi \in U_{n}\right)\right.
$$

Note that, for $\pi \in U_{n}, \pi \sigma_{i+1} \in U_{n}$ iff either $i \in\{0, n-2\}$ or $\phi(\pi)_{i+1} \neq \phi(\pi)_{i+2}$.

Observation 3.5 For every $\pi \in U_{n}$ and $1 \leqslant j \leqslant n-1$,

$$
\begin{gathered}
\phi\left(\rho_{0}(\pi)\right)_{j}=\left\{\begin{array}{lll}
\phi(\pi)_{1}-1 & (\bmod n), & \text { if } j=1 \text { and } \phi(\pi)_{2}=0 ; \\
\phi(\pi)_{1}+1 & (\bmod n), & \text { if } j=1 \text { and } \phi(\pi)_{2}=1 ; \\
\phi(\pi)_{2}+1 & (\bmod 2), & \text { if } j=2 ; \\
\phi(\pi)_{j}, & \text { if } j \neq 1,2,
\end{array}\right. \\
\phi\left(\rho_{i}(\pi)\right)_{j}=\phi(\pi)_{\sigma_{i+1}(j)} \quad(1 \leqslant i \leqslant n-1, \forall j)
\end{gathered}
$$

and

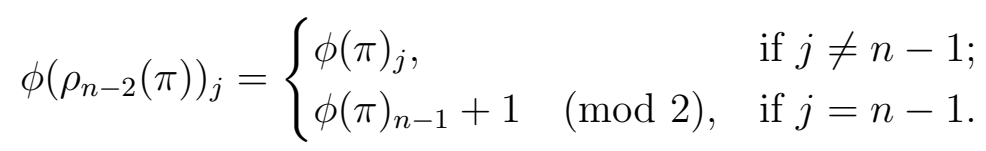

Proposition 3.6 The maps $\rho_{i}$, when extended multiplicatively, determine a well defined transitive $\widetilde{C}_{n-2}$-action on the set $U_{n}$ of arc permutations.

Proof. To prove that the operation is a $\widetilde{C}_{n-2}$-action, it suffices to show that it is consistent with the Coxeter relations defining $\widetilde{C}_{n-2}$ when the operator $\rho_{i}$ is interpreted as an action of the generator $s_{i}$. All relations may be easily verified using Observation 3.5; we leave the details to the reader.

To prove that the action is transitive, notice first that $\rho_{0}(\pi)(1)=\pi(2)=$ $\pi(1) \pm 1(\bmod n)$. It thus suffices to prove that, for every $1 \leqslant k \leqslant n$, the maximal parabolic subgroup $\left\langle s_{1}, \ldots, s_{n-2}\right\rangle$ of $\widetilde{C}_{n-2}$ acts transitively on the set $U_{n}^{(k)}:=\left\{\pi \in U_{n}\right.$ : $\pi(1)=k\}$. Indeed, this parabolic subgroup is isomorphic to the classical Weyl group $B_{n-2}$. By Observation 3.5, the restricted $B_{n-2}$-action on $U_{n}^{(k)}$ may be identified with the natural $B_{n-2}$-action on all subsets of $\{1, \ldots, n-2\}$, and is thus transitive. 


\section{A Graphic Hyperplane Arrangement}

\subsection{Real Hyperplane Arrangements}

Let $\mathcal{A}$ be an arrangement of finitely many linear hyperplanes in $\mathbb{R}^{d}$ that is central and essential, meaning that $\cap_{H \in \mathcal{A}} H=\{0\}$. Let $L=\sqcup_{i=0}^{d} L_{i}$ be the corresponding graded poset of intersection subspaces, ordered by reverse inclusion. $L$ is a geometric lattice.

Let $\mathcal{C}$ be the set of chambers of $\mathcal{A}$, namely the connected components of the complement $\mathbb{R}^{d} \backslash \cup_{H \in \mathcal{A}} H$. Define a graph structure $G_{1}(\mathcal{A})$ on the set of vertices $\mathcal{C}$, with two chambers $c, c^{\prime} \in \mathcal{C}$ connected by an edge if they are separated by exactly one hyperplane in $\mathcal{A}$. It is well-known that the diameter of this graph is equal to the number of hyperplanes, $\left|L_{1}\right|=|\mathcal{A}|$.

The reflection arrangement $\mathcal{A}_{n-1}$ of type $A_{n-1}$, corresponding to the symmetric group $S_{n}$, has as ambient space the $(n-1)$-dimensional subspace

$$
W=\left\{\bar{x}=\left(x_{1}, \ldots, x_{n}\right) \in \mathbb{R}^{n} \mid x_{1}+\ldots+x_{n}=0\right\}
$$

of $\mathbb{R}^{n}$. Its hyperplanes are $H_{i j}:=\left\{\bar{x} \in W \mid x_{i}=x_{j}\right\}$ for $1 \leqslant i<j \leqslant n$. The chambers may be identified with permutations in $S_{n}$, via

$$
c_{\pi}:=\left\{\bar{x} \in W \mid x_{\pi(1)}<x_{\pi(2)}<\cdots<x_{\pi(n)}\right\} \quad\left(\forall \pi \in S_{n}\right) .
$$

The symmetric group $S_{n}$ acts on the chambers via $\sigma_{i}\left(c_{\pi}\right):=c_{\pi \sigma_{i}}$, the unique chamber which is separated from $c_{\pi}$ only by the hyperplane $H_{\pi(i), \pi(i+1)}$. Then, for every $\pi \in S_{n}, c_{\pi}$ and $-c_{\pi}=c_{\pi w_{0}}$ are antipodes in the graph $G_{1}\left(\mathcal{A}_{n-1}\right)$, where $w_{0}:=[n, n-1, \ldots, 1]$ is the longest element in $S_{n}$.

A (simple undirected) graph $G=(V, E)$ of order $n$ consists of a set $V=\left\{v_{1}, \ldots, v_{n}\right\}$ of vertices and a set $E$ of edges, which are unordered pairs of distinct vertices. The associated graphic arrangement $\mathcal{A}(G)$ is the hyperplane arrangement in $W \cong \mathbb{R}^{n-1}$ defined by

$$
\mathcal{A}(G):=\left\{H_{i j} \mid\left\{v_{i}, v_{j}\right\} \in E\right\} \subseteq \mathcal{A}_{n-1} .
$$

For example, if $K_{n}$ be the complete graph of order $n$ then the associated graphic arrangement $\mathcal{A}\left(K_{n}\right)$ is the whole reflection arrangement $\mathcal{A}_{n-1}$. For more information see [8].

\subsection{The Graph of Chambers $G_{1}\left(U_{n}^{\prime}\right)$}

Definition 4.1 Let $G$ be a graph of order $n$. Two permutations $\pi, \tau \in S_{n}$ are $G$ equivalent if the points $(\pi(1), \ldots, \pi(n)),(\tau(1), \ldots, \tau(n)) \in W$ lie in the same chamber of the associated graphic arrangement $\mathcal{A}(G)$.

Index by $1, \ldots, n$ the vertices of the complete graph $K_{n}$, and consider the graph $K_{n}^{\prime}$ obtained by deleting the edges $\{1,2\},\{2,3\}, \ldots,\{n-1, n\}$ and $\{n, 1\}$ from $K_{n}$. Let $\mathcal{A}_{n-1}^{\prime}:=\mathcal{A}\left(K_{n}^{\prime}\right)$ be the associated graphic arrangement. Two permutations $\pi, \tau \in S_{n}$ 
are $K_{n}^{\prime}$-equivalent if and only if there exist permutations $\pi=\pi_{0}, \pi_{1}, \ldots, \pi_{t}=\tau$ such that, for every $0 \leqslant r \leqslant t-1$, there exists $1 \leqslant i \leqslant n-1$ such that $\pi_{r+1}=\pi_{r} \sigma_{i}$ and $\pi_{r} \sigma_{i} \pi_{r}^{-1} \in\left\{\sigma_{j}: 1 \leqslant j \leqslant n-1\right\} \cup\{(1, n)\}$. In other words, the $K_{n}^{\prime}$-equivalence is the transitive closure of the the following relation: there exist $1 \leqslant j<n$ such that the letters $j$ and $j+1$, or 1 and $n$, are adjacent in $\pi$, and $\tau$ is obtained from $\pi$ by switching their positions.

Remark 4.2 Since $K_{n}^{\prime}$ is invariant under the natural action of the dihedral group $I_{2}(n)$, this group may be embedded in the automorphism group of the graph $G_{1}\left(\mathcal{A}_{n-1}^{\prime}\right)$. Indeed, let $\gamma$ be the cycle $(1,2, \ldots, n) \in S_{n}$ and $w_{0}:=[n, n-1, \ldots, 1]$ the longest element in $S_{n}$. If $A$ is a $K_{n}^{\prime}$-equivalence class then for $0 \leqslant j<n$ and $\epsilon \in\{0,1\}, w_{0}^{\epsilon} \gamma^{j} A$ is a also $K_{n^{-}}^{\prime}$ equivalence class. Moreover, edges in $G_{1}\left(\mathcal{A}_{n-1}^{\prime}\right)$ are indexed by pairs of $K_{n}^{\prime}$-equivalence classes, where for every such a pair, $(A, B)$ is an edge in $G_{1}\left(\mathcal{A}_{n-1}^{\prime}\right)$ if and only if $w_{0}^{\epsilon} \gamma^{j}(A, B)$ is an edge in $G_{1}\left(\mathcal{A}_{n-1}^{\prime}\right)$.

Definition 4.3 (i) Define $\tilde{K}_{n}^{\prime}$-equivalence on the subset of arc permutations $U_{n} \subset S_{n}$ as the transitive closure of the relation: there exist $1 \leqslant j<n$ such that the letters $j$ and $j+1$, or 1 and $n$, are adjacent in $\pi$, and $\tau$ is obtained from $\pi$ by switching their positions.

(ii) Let $U_{n}^{\prime}$ be the set of $\tilde{K}_{n}^{\prime}$-equivalence classes in $U_{n}$.

(iii) Let $G_{1}\left(U_{n}^{\prime}\right)$ be the graph whose vertex set is $U_{n}^{\prime}$; two $\tilde{K}_{n}^{\prime}$-equivalence classes in $U_{n}^{\prime}$ are adjacent in $G_{1}\left(U_{n}^{\prime}\right)$ if they have representatives, whose corresponding chambers in $G_{1}\left(\mathcal{A}_{n-1}\right)$ lie in adjacent chambers in $G_{1}\left(\mathcal{A}_{n-1}^{\prime}\right)$.

Observation 4.4 For $n>3$ all $\tilde{K}_{n}^{\prime}$-equivalence classes in $U_{n}$ consist of four permutations $\left\{\pi, \pi \sigma_{1}, \pi \sigma_{n-1}, \pi \sigma_{1} \sigma_{n-1}\right\}$.

Proof. For every $\pi \in U_{n}$ and $1<i<n-2$, if $\pi(i+1)=\pi(i) \pm 1$ then $\pi \sigma_{i} \notin U_{n}$. On the other hand, for every $\pi \in U_{n}$ and $i \in\{1, n-1\}, \pi \sigma_{i} \in U_{n}$.

Note that, by definition, two $\tilde{K}_{n}^{\prime}$-equivalent arc permutations are $K_{n}^{\prime}$-equivalent in $S_{n}$; hence, they lie in same chamber in $G_{1}\left(\mathcal{A}_{n-1}^{\prime}\right)$. One concludes that $G_{1}\left(U_{n}^{\prime}\right)$ contains no loops.

\section{Example 4.5}

(a) For $n=4$ there are four $K_{4}^{\prime}$-equivalence classes in $S_{4}$ :

$\mathbf{1 2 3 4}=\{[1234],[1324],[2134],[1243],[2143],[2413]\}$ and its images under cyclic rotations $\gamma^{j} \mathbf{1 2 3 4}, 0 \leqslant j<4$.

The edges in the graph $G_{1}\left(\mathcal{A}_{3}^{\prime}\right)$ are all cyclic rotations of $(\mathbf{1 2 3 4}, \mathbf{2 3 4 1})$, thus the graph is a 4-cycle. Since, each $K_{4}^{\prime}$-equivalence class contains one $\tilde{K}_{4}^{\prime}$-class in $U_{4}^{\prime}$, the graphs $G_{1}\left(\mathcal{A}_{3}^{\prime}\right)$ and $G_{1}\left(U_{4}^{\prime}\right)$ are identical. 
(b) For $n=5$ there are three types of $K_{4}^{\prime}$-equivalence classes in $S_{5}$ :

$\mathbf{1 2 3 4 5}=\{[12345],[13245],[21345],[12435],[21435],[12354],[13254],[21354]\}$ and its ten images under dihedral group action $w_{0}^{\epsilon} \gamma^{j} \mathbf{1 2 3 4 5}, 0 \leqslant j<5, \epsilon \in\{0,1\}$;

$13452=\{[13452],[14352],[13542]\}$ and its ten images under dihedral group action;

$\mathbf{1 3 5 2 4}=\{[13524]\}$ and its ten images under dihedral group action.

The edges in the graph $G_{1}\left(\mathcal{A}_{4}^{\prime}\right)$ are

$\{(12345,32154),(12345,13524),(12345,24135),(12345,14325)$,

$(\mathbf{1 3 5 2 4}, \mathbf{1 3 4 5 2}),(\mathbf{1 3 5 2 4}, \mathbf{3 5 1 2 4}),(\mathbf{1 3 4 5 2}, \mathbf{1 4 3 2 5})\}$ and their images under the dihedral group action.

There are ten $\tilde{K}_{4}^{\prime}$-equivalence classes in $U_{4}$, each contained in one of the images under the dihedral group action of $\mathbf{1 2 3 4 5}$. Thus the graph $G_{1}\left(U_{5}^{\prime}\right)$ is a 10-cycle.

\section{Stanley's Conjecture}

It was conjectured by Richard Stanley [12] that all diagonals are flipped in a geodesic between two antipodes in the flip graph $\Gamma_{n}$ of colored triangle-free triangulations. A bijection between the set of triangle-free triangulations in $C T F T(n)$ and the subset $U_{n}^{\prime}$ of chambers in the graphic hyperplane arrangement $\mathcal{A}\left(K_{n}^{\prime}\right)$, which preserves the underlying graph structure, is applied to prove Stanley's conjecture for distinguished pairs of antipodes.

Theorem 5.1 The flip graph $\Gamma_{n}$ (without edge labeling) is isomorphic to the graph of chambers $G_{1}\left(U_{n}^{\prime}\right)$.

Furthermore,

Theorem 5.2 There exists an edge-orientation of the flip graph $\Gamma_{n}$ such that, for any oriented edge of adjacent triangulations $(T, S), S$ is obtained from $T$ by flipping the diagonal $[i, j]$ if and only if the corresponding chambers are separated by the hyperplane $x_{i}=x_{j}$.

It follows that Stanley's conjecture holds for distinguished pairs of antipodes.

Corollary 5.3 For every colored triangle-free triangulation $T \in C T F T(n)$, every diagonal is flipped exactly once along the shortest path from $T$ to same triangulation with reversed coloring $T^{R}$. 


\section{$6 \quad$ Proof of Theorem 5.1}

\subsection{A $\widetilde{C}_{n-4}$-Action on $U_{n}^{\prime}$}

For every $\pi \in U_{n}$ denote the $K_{n}^{\prime}$-class of $\pi$ in $U_{n}$ by $\bar{\pi}$. By Observation 4.4 , for every $\pi \in$ $U_{n}, \bar{\pi} \in U_{n}^{\prime}$ may be represented by a series of $n-2$ subsets: $\{\pi(1), \pi(2)\},\{\pi(3)\}, \ldots,\{\pi(n-$ $2)\},\{\pi(n-1), \pi(n)\}$, where all subsets except of the first and the last are singletons.

For every simple reflection $\sigma_{i} \in S_{n-2}, 1<i<n-3$, let $\bar{\pi} \sigma_{i}$ be the series of subsets obtained from $\bar{\pi}$ by replacing the letters in the $i$-th and $i+1$-st subsets. Let $\bar{\pi} \sigma_{1}$ be obtained from $\bar{\pi}$ by replacing letters in the first two subsets as follows: if $\{\pi(1), \pi(2)\}=$ $\{\pi(3)-2, \pi(3)-1\}$ then the first two subsets in $\bar{\pi} \sigma_{1}$ are $\{\pi(3), \pi(3)-1\},\{\pi(3)-2\}$; $\{\pi(1), \pi(2)\}=\{\pi(3)+1, \pi(3)+2\}$ then the first two subsets in $\bar{\pi} \sigma_{1}$ are $\{\pi(3), \pi(3)+$ $1\},\{\pi(3)+2\}$. Similarly, $\bar{\pi} \sigma_{n-3}$ is obtained from $\bar{\pi}$ by replacing the letter in the $n-3$-rd subset with $\pi(n-2)-2$ if $\{\pi(n-1), \pi(n)\}=\{\pi(n-2)-2, \pi(n-2)-1\}$ and with $\pi(n+2)$ otherwise.

For every $0 \leqslant i \leqslant n$ let $\theta_{i}: U_{n}^{\prime} \mapsto U_{n}^{\prime}$ be

$$
\theta_{i}(\bar{\pi})=\left\{\begin{array}{ll}
\bar{\pi} \sigma_{i+1}, & \text { if } \bar{\pi} \sigma_{i+1} \in U_{n}^{\prime}, \\
\bar{\pi}, & \text { if } \bar{\pi} \sigma_{i+1} \notin U_{n}^{\prime} .
\end{array} \quad\left(\forall \bar{\pi} \in U_{n}^{\prime}\right)\right.
$$

Observation 6.1 The maps $\theta_{i},(0 \leqslant i \leqslant n-4)$, when extended multiplicatively, determine a well defined transitive $\widetilde{C}_{n-4}$-action on $U_{n}^{\prime}$.

Proof is similar to the proof of Observation 3.6 and is omitted.

Observation 6.2 Two chambers in $\bar{\pi}, \bar{\tau} \in U_{n}^{\prime}$ are adjacent in $G_{1}\left(U_{n}^{\prime}\right)$ if and only if there exist $0 \leqslant i \leqslant n-4$, such that $\theta_{i}(\bar{\pi})=\bar{\tau}$.

\subsection{A Graph Isomorphism}

Let $f: C T F T(n) \mapsto U_{n}^{\prime}$ be defined as follows: if $[a, a+2]$ is the short chord labeled 0 then let $\{\pi(1), \pi(2)\}=\{a, a+1\}$. For $0<i<n-4$, assume that the chord labeled $i-1$ in $T$ is $[a-k, a+m]$ for some $k, m \geqslant 1, k+m=i+1$. The chord labeled $i$ is then either $[a-k-1, a+m]$ or $[a-k, a+m+1]$. Let $i+1$-st subset be $\{a-k-1\}$ in the former case and $\{a+m\}$ in the latter. Finally, let the last subset consist of the remaining two letters.

Claim 6.3 The map $f: C T F T(n) \mapsto U_{n}^{\prime}$ is a bijection.

Proof. The map $f$ is invertible.

Recall the definition of $T^{R}$ from Section 2 .

Observation 6.4 For every $T \in C T F T(n), f\left(T^{R}\right)$ is obtained from $f(T)$ by reversing the order of the subsets. 
Recall from Subsection 2.2 the affine Weyl group $\widetilde{C}_{n-4}$-action on $C T F T(n)$.

To complete the proof of Theorem 5.1 it suffices to show that

Proposition 6.5 For every Coxeter generator $s_{i}$ of $\widetilde{C}_{n-4}(0 \leqslant i \leqslant n-4)$ and $T \in$ $C T F T(n)$

$$
f\left(s_{i} T\right)=s_{i} f(T)
$$

where $s_{i} f(T):=\theta_{i}(f(T))$.

Proof. For $i=0$, let $[a, a+2]$ be the short chord labeled 0 in $T$. Then the chord labeled 1 is either $[a, a+3]$ or $[a-1, a+2]$. In the first case the short chord labeled 0 in $s_{0} T$ is $[a+1, a+3]$ and all other chords are unchanged, in particular, the chord labeled 1 in $s_{0} T$ is $[a, a+3]$. By definition of the map $f$, the first two subsets in $f(T)$ are $\{a, a+1\},\{a+2\}$ and the first two subsets in $f\left(s_{0} T\right)$ are $\{a+1, a+2\},\{a\}$ and the rest are not changed. On the other hand, by definition of $\theta_{0}$, the first two subsets in $s_{0} f(T)$ are $\{a+1, a+2\},\{a\}$ and the rest are unchanged. A similar analysis shows that $f\left(s_{0} T\right)=s_{0} f(T)$ when the chord labeled 1 is $[a-1, a+2]$.

For $0<i<n-4$ let the chord labeled $i-1$ in $T$ be $[a-k, a+m]$ for some $k, m \geqslant 1$, $k+m=i+1$. The chords labeled $i$ and $i+1$ are then either $[a-k-1, a+m],[a-k-2, a+m]$ respectively, or $[a-k, a+m+1],[a-k, a+m+2]$ or $[a-k-1, a+m],[a-k-1, a+m+1]$ or $[a-k, a+m+1],[a-k-1, a+m+1]$. In the first two cases $s_{i} T=T$, so $f\left(s_{i} T\right)=f(T)$. On the other hand, in these cases $f(T) \sigma_{i} \notin U_{n}^{\prime}$, so $s_{i} f(T)=f(T)$.

If the chords labeled $i$ and $i+1$ in $T$ are $[a-k-1, a+m],[a-k-1, a+m+1]$ respectively, then the chords labeled $i$ and $i+1$ in $s_{i} T$ are $[a-k, a+m+1],[a-k-1, a+m+1]$. So, the $i$-th and $i+1$-st subsets in $f(T)$ are $\{a-k-1\},\{a+m\}$, and they are switched in $s_{i} f(T)$, so same as the corresponding subsets in $f\left(s_{i} T\right)$. The proof of the forth case is similar.

Finally, by Observation 6.4, $f\left(s_{0} T\right)=s_{0} f(T)$ implies that $f\left(s_{n-4} T\right)=s_{n-4} f(T)$.

\section{$7 \quad$ Proof of Theorem 5.2}

\subsection{Orienting the Colored Flip Graph}

The goal of this subsection is to equip the colored flip graph $\Gamma_{n}$ with an edge orientation that will be used to encode the location of the flipped diagonals. It will be proved later that this orientation satisfies the conditions of Theorem 5.2. Our starting point is the edge labeling, mentioned in Section 2, which encodes the order of the chords.

Recall from [1] the bijection

$$
\varphi: C T F T(n) \rightarrow \mathbb{Z}_{n} \times \mathbb{Z}_{2}^{n-4}
$$


defined as follows: Let $T \in C T F T(n)$. If the (short) chord labeled 0 in $T$ is $[a-1, a+1]$ for $a \in \mathbb{Z}_{n}$, let $\varphi(T)_{0}:=a$. For $1 \leqslant i \leqslant n-4$, assume that the chord labeled $i-1$ in $T$ is $[a-k, a+m]$ for some $k, m \geqslant 1, k+m=i+1$. The chord labeled $i$ is then either $[a-k-1, a+m]$ or $[a-k, a+m+1]$. Let $\varphi(T)_{i}$ be 0 in the former case and 1 in the latter.

By definition of the map $\varphi$,

Claim 7.1 For every vector $v=\left(v_{0}, \ldots, v_{n-4}\right) \in \mathbb{Z}_{n} \times \mathbb{Z}_{2}^{n-4}$ and every $0 \leqslant i \leqslant n-4$, the diagonal labeled $i$ in the triangulation $T=\varphi^{-1}(v)$ is $[k, m]$ where

$$
k:=v_{0}-1-i+\sum_{j=1}^{i} v_{i} \in \mathbb{Z}_{n}
$$

and

$$
m:=v_{0}+1+\sum_{j=1}^{i} v_{i} \in \mathbb{Z}_{n}
$$

Here $0,1 \in \mathbb{Z}_{2}$ are interpreted as $0,1 \in \mathbb{Z}_{n}$.

It follows that

Corollary 7.2 [1, Lemma 5.7] For every $T \in \operatorname{CTFT}(n)$, if $\varphi(T)=\left(v_{0}, \ldots, v_{n}\right)$ then $\varphi\left(T^{R}\right)_{0}=2+\sum_{i=0}^{n-4} v_{i} \in \mathbb{Z}_{n}$ and $\varphi\left(T^{R}\right)_{i}=1-v_{n-3-i} \in \mathbb{Z}_{2}(1 \leqslant i \leqslant n-4)$.

Observation 7.3 [1, Observation 3.1] For every $T \in C T F T(n)$ and a Coxeter generator $s_{i}$ of $\widetilde{C}_{n-4}$

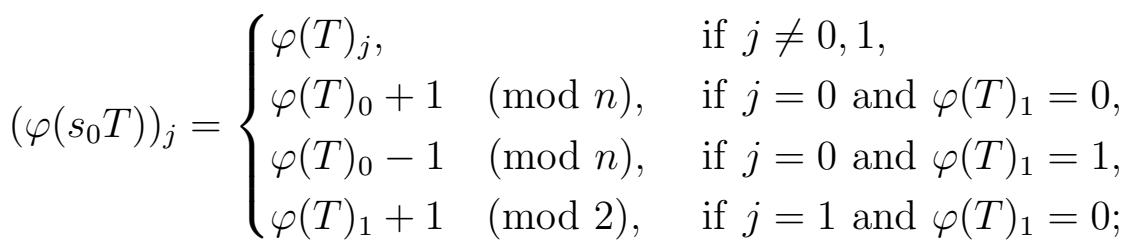

$$
\begin{aligned}
& \left(\varphi\left(s_{n-4} T\right)\right)_{j}= \begin{cases}\varphi(T)_{j}, & \text { if } j \neq n, \\
\varphi(T)_{n}+1 \quad(\bmod 2), & \text { if } j=n ;\end{cases}
\end{aligned}
$$

and

$$
\left(\varphi\left(s_{i} T\right)\right)_{j}=\varphi(T)_{\sigma_{i}(j)} \quad(0<i<n-4) ;
$$

where $\sigma_{i}:=(i, i+1)$ the adjacent transposition.

We use this observation to orient the edges in $\Gamma_{n}$. 
Definition 7.4 Orient the edges in $\Gamma_{n}$ as follows: If the diagonal labeled $n-4$ is flipped orient the corresponding edge from the triangulation encoded by last entry 0 to the one with last entry 1 . If the flip is of the diagonal labeled $0<i<n-4$ orient the edge from $T$ with $\varphi(T)_{i}=0, \varphi(T)_{i+1}=1$ to the one with these two entries switched; if it flips the diagonal labeled 0 orient it by the first entry from $T$ with $\varphi(T)_{0}=j$ to the one with first entry under $\varphi$ being $j+1$.

See Figure 7.1 for the orientation of $\Gamma_{6}$, where each colored triangulation $T$ is labeled by the vector $\varphi(T)$.

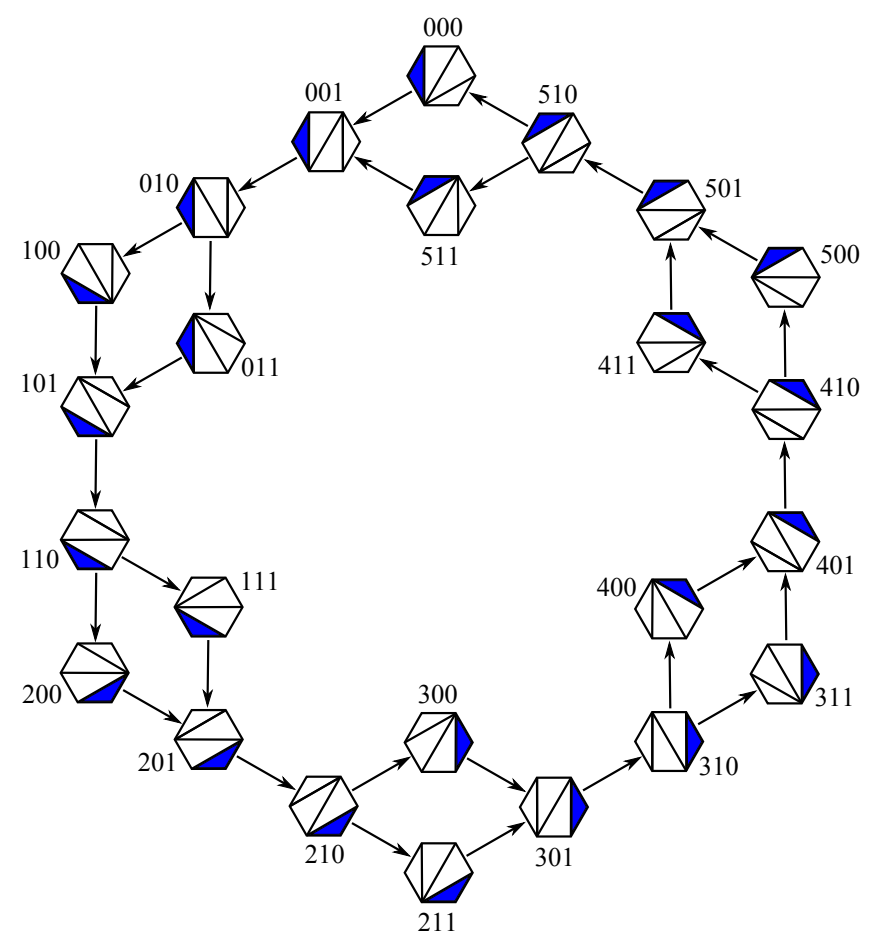

Figure 2: $\Gamma_{6}$ with orientation

Lemma 7.5 For every $T \in C T F T(n)$, the orientation of the edges along any geodesic from $T$ to $T^{R}$ is coherent with the orientation of $\Gamma_{n}$ described in Definition 7.4 ; namely, all edges in a geodesic have the same orientation as in the oriented $\Gamma_{n}$ or all have the opposite orientation.

Proof. Consider the dominance order on vectors in $\mathbb{Z}_{n} \times \mathbb{Z}_{2}^{n-4}$; namely,

$$
\left(v_{0}, \ldots, v_{n-4}\right) \leqslant\left(u_{0}, \ldots, u_{n-4}\right)
$$

if and only if

$$
\sum_{i=0}^{k} v_{i} \leqslant \sum_{i=0}^{k} u_{i} \quad(0 \leqslant k \leqslant n-4),
$$


where $0, \ldots, n-1 \in \mathbb{Z}_{n}$ are interpreted as $0, \ldots, n-1 \in \mathbb{Z}$, and similarly for $\mathbb{Z}_{2}$.

The resulting poset is ranked by

$$
\ell\left(v_{0}, \ldots, v_{n-4}\right):=\sum_{i=0}^{n-4}(n-3-i) v_{i}
$$

Using Corollary 7.2, the reader can verify that for every $T \in C T F T(n)$

$$
\ell\left(\varphi\left(T^{R}\right)\right)-\ell(\varphi(T)) \equiv n(n-3) / 2 \quad(\bmod n(n-3)),
$$

which is the distance between $T$ and $T^{R}$ (Theorem 2.5).

Finally, notice that for every edge $e=\left(S_{1}, S_{2}\right)$ in $\Gamma_{n}$, the edge $e$ is oriented from $S_{1}$ to $S_{2}$ if and only if

$$
\ell\left(\varphi\left(S_{2}\right)\right)-\ell\left(\varphi\left(S_{1}\right)\right) \equiv 1 \quad(\bmod n(n-3))
$$

One concludes that either all steps in a geodesic increase the rank function by one modulo $n(n-3)$ or all steps decrease it by one. Hence the lemma holds.

We note that this proof essentially appears (implicitly) in [1], where an algebraic interpretation of the rank function as a length function on $\widetilde{C}_{n-4}$ is given; see, in particular, [1, Sections 3.3 and 5.2].

Now color each edge $\left(S_{1}, S_{2}\right)$ of $\Gamma_{n}$, oriented from $S_{1}$ to $S_{2}$, by the chord $[i, j]$ which is erased from $S_{1}$. Ignore the edge-orientation and let $\hat{\Gamma}_{n}$ be the resulting edge-labeled flip graph.

\subsection{Edge-Colored Graph Isomorphism}

Consider an edge-labeled version of the graph $G_{1}\left(U_{n}^{\prime}\right)$, denoted by $\hat{G}_{1}\left(U_{n}^{\prime}\right)$, where the edge between two adjacent chambers is labeled by the separating hyperplane.

Theorem 7.6 The edge-labeled graphs $\hat{G}_{1}\left(U_{n}^{\prime}\right)$ and $\hat{\Gamma}_{n}$ are isomorphic.

Note that this theorem implies Theorem 5.2.

\section{Proof.}

By Observation 6.2, two chambers $\bar{\pi}, \bar{\tau} \in U_{n}^{\prime}$ are adjacent in $G_{1}\left(U_{n}^{\prime}\right)$ if and only if there exist corresponding arc permutations $\pi, \tau \in U_{n}$ and $1<i<n-1$, such that $\pi \sigma_{i}=\tau$. The separating hyperplane is then $x_{k}=x_{m}$ if and only if $(k, m) \pi=\tau$, or equivalently $\pi \sigma_{i} \pi^{-1}=(k, m)$, for the transposition $(k, m) \in S_{n}$.

Recall the bijection $f: C T F T(n) \mapsto U_{n}^{\prime}$, defined in Subsection 6.2. Since $f$ induces a graph isomorphism, for every $1<i<n-1$, if $\pi, \pi \sigma_{i}$ are two arc permutations then $\left.f^{-1}(\bar{\pi}), f^{-1}\left(\overline{\pi \sigma_{i}}\right)\right)$ forms an edge in $\Gamma_{n}$. In order to prove Theorem 5.2, it suffices to show 
that $\left.f^{-1}\left(\bar{\pi} \sigma_{i}\right)\right)$ is obtained from $f^{-1}(\bar{\pi})$ by flipping the diagonal $[k, m]$, when the edge is oriented from $f^{-1}(\bar{\pi})$ to $f^{-1}\left(\bar{\pi} \sigma_{i}\right)$.

Indeed, an edge is oriented from $f^{-1}(\bar{\pi})$ to $f^{-1}\left(\overline{\pi \sigma_{1}}\right)$ if and only if the latter triangulation is obtained from the first by flipping the short chord labeled $0[a-1, a+1]$; namely, by replacing the diagonal $[a-1, a+1]$ by $[a, a+2]$, where the diagonal labeled 1 is $[a-1, a+2]$. By definition of the map $f$ the first two subsets in $\bar{\pi}$ are $\{a-1, a\},\{a+1\}$ and in $\bar{\pi} \sigma_{1}$ : $\{a, a+1\},\{a-1\}$. Letting $\pi=[a, a-1, a+1, \ldots]$ one gets $\pi \sigma_{1} \pi^{-1}=(a-1, a+1)$, so the separating hyperplane is $x_{a-1}=x_{a+1}$.

For $1<i<n-3$, an edge is oriented from $f^{-1}(\bar{\pi})$ to $f^{-1}\left(\overline{\pi \sigma_{i}}\right)$ if and only if the chord labeled $i-1$ is $[a-k, a+m]$ and the latter triangulation is obtained from the first by flipping a diagonal $[a-k-1, a+m]$; namely, by replacing the diagonal $[a-k-1, a+m]$ by $[a-k, a+m+1]$. Then the $i$-th and $i+1$-st subsets in $\bar{\pi}$, which are $\{a-k-1\},\{a+m\}$, are switched in $\overline{\pi \sigma_{i+1}}$. So $\pi(i)=a-k-1$ and $\pi(i+1)=a+m+1$, and $\pi \sigma_{i+1} \pi^{-1}=$ $(a-k-1, a+m)$.

Finally, an edge is oriented from $f^{-1}(\bar{\pi})$ to $f^{-1}\left(\overline{\pi \sigma_{n-3}}\right)$ if and only if the chord labeled $n-5$ is $[b-2, b+1]$ and the latter triangulation is obtained from the first by flipping the short chord labeled $n-4[b-1, b+1]$; namely, by replacing the diagonal $[b-1, b+1]$ by $[b, b+2]$. Then the last two subsets in $\bar{\pi}$ are $\{b-1\},\{b, b+1\}$ and in $\overline{\pi \sigma_{n-3}}:\{b\},\{b-1, b\}$. So $\pi=[\ldots, b-1, b+1, b]$ one gets $\pi \sigma_{n-3} \pi^{-1}=[b-1, b+1]$.

\section{$8 \quad$ Proof of Corollary 5.3}

Recall that $T$ and $T^{R}$ are antipodes (Theorem 2.5).

Proposition 8.1 For every colored triangle-free triangulation $T \in C T F T(n)$, the corresponding chambers in $\mathcal{A}\left(K_{n}^{\prime}\right)$ satisfy

$$
c_{f\left(T^{R}\right)}=-c_{f(T)} .
$$

Proof. Let $w_{0}:=[n, n-1, n-2, \ldots 1]$ be the longest permutation in $S_{n}$. It follows from Observation 6.4 that for every $\pi \in U_{n}$

$$
\left(f^{-1}(\bar{\pi})\right)^{R}=f^{-1}\left(\overline{\pi w_{0}}\right) .
$$

Notice that the points $\pi$ and $\pi w_{0}$ in $\mathbb{R}^{n}$ belong to negative chambers $c$ and $-c$. The proof is completed.

Proof of Corollary 5.3. By Proposition 8.1, the set of hyperplanes which separate the chamber $c_{f(T)}$ from the chamber $c_{f\left(T^{R}\right)}$ is the set of all hyperplanes in $\mathcal{A}\left(K_{n}^{\prime}\right)$. By Theorem 7.6 together with Lemma 7.5, one deduces that all diagonals have to be flipped 
at least once in a geodesic from $T$ to $T^{R}$. Finally, by Theorems 2.4 and 2.5, the distance between $T$ and $T^{R}$ in $\Gamma_{n}$ is equal to the number of diagonals in a convex $n$-gon. Hence, each diagonal is flipped exactly once.

\section{Geodesics and Shifted Tableaux}

Let $T_{0}$ be the canonical colored star triangle-free triangulation; that is the triangulation, which consists of the chords $[0,2],[0,3], \ldots,[0, n-2]$ labeled $0, \ldots, n-4$ respectively.

\subsection{Order on the Diagonals}

By Corollary 5.3, every geodesic from the canonical colored star traingle-free triangulation $T_{0}$ to $T_{0}^{R}$ determines a linear order on the diagonals. The following theorem characterizes these linear orders.

Theorem 9.1 An order on the set of diagonals $\{[i, j]: 1 \leqslant i<j-1 \leqslant n-1\}$ of a convex $n$-gon appears in geodesics in $\Gamma_{n}$ from $T_{0}$ to its reverse $T_{0}^{R}$ if and only if it is a linear extension of the coordinate-wise order with respect to the natural order

$$
0<1<2<\cdots<n-1
$$

or its reverse

$$
0 \equiv n<n-1<n-2<\cdots<1
$$

Proof. Clearly, every geodesic from $T_{0}$ to $T_{0}^{R}$ starts with either flipping $[0,2]$ or $[0, n-2]$. By symmetry, exactly half start by flipping [0,2].

First, we will prove that an order on the set of diagonals of a convex $n$-gon corresponding to geodesics from $T_{0}$ to $T_{0}^{R}$, which start by flipping [0,2], is a linear extension of the coordinate-wise order with respect to the natural order $0<1<2<\cdots<n-1$.

Recall that by Corollary 5.3, every hyperplane is not crossed more than once. Thus, in order to prove this, it suffices to show that in every gallery from the identity chamber

$c_{[0,1,2, \ldots, n-1]}$ to its negative, that start by crossing the hyperplane $H_{0,2}$, the hyperplane $H_{k, l}$ is crossed after the hyperplane $H_{i, j}$, whenever $i+1<j, k+1<l$, and $(i, j)<(k, l)$ in point-wise coordinate order. In other words, it suffices to prove that for every arc permutation $\pi \in U_{n}$, if $\bar{\pi} \in U_{n}^{\prime}$ corresponds to a chamber in such a gallery then $\pi^{-1}(i)<$ $\pi^{-1}(j) \Longrightarrow \pi^{-1}(k)<\pi^{-1}(l)$.

Clearly, this holds for the arc permutations which correspond to the identity chamber $c_{[0,1, \ldots, n-1]}$ and to its negative $-c_{[0,1, \ldots, n-1]}=c_{[n-1, n-2, \ldots, 0]}$. With regard to all other chambers in these galleries, notice first, that all geodesics from $T_{0}$ to $T_{0}^{R}$ must end by flipping either $[n-3, n-1]$ or $[1,3]$. Let $S \in C T F T(n)$ be the triangulation, which consists of the chords $[1,3],[0,3],[0,4], \ldots,[0, n-2]$ labeled $0,1, \ldots, n-4$ respectively. 
Then $T_{0}^{R}$ is obtained from $S^{R}$ by flipping $[1,3]$ and $S$ is obtained from $T_{0}$ by flipping [0,2]. Since $S$ and $S^{R}$ are antipodes, it follows that $S$ does not appear in a geodesic from $T_{0}$ to $T_{0}^{R}$ which start by flipping $[0,2]$. Thus every geodesic from $T_{0}$ to $T_{0}^{R}$, which start by flipping $[0,2]$ must end by flipping $[n-3, n-1]$. One concludes that for every $\pi \in U_{n}$, if $\bar{\pi} \in U_{n}^{\prime}$ is a chamber in such a gallery which is not first or last, then $\pi^{-1}(2)<\pi^{-1}(0)$ and $\pi^{-1}(n-3)<\pi^{-1}(n-1)$. Thus the first letter in $\pi, \pi(0)$, is not 0 or $n-1$. There are three cases to analyze:

If $\pi(0)=1$ then, since $\pi^{-1}(n-3)<\pi^{-1}(n-1)$ and $\pi$ is an arc permutation, $0=$ $\left.\pi^{-1}(1)<\pi^{-1}(2)<\cdots<\pi^{-1}(n-3)<\pi^{-1}(n-1)\right)$.

If $\pi(0)=n-2$ then, since $\pi^{-1}(2)<\pi^{-1}(0)$ and $\pi$ is an arc permutation, $0=$ $\pi^{-1}(n-2)<\pi^{-1}(n-3)<\cdots<\pi^{-1}(2)<\pi^{-1}(0)$.

Finally, if $2 \leqslant \pi(0) \leqslant n-3$ then, since $\pi^{-1}(n-3)<\pi^{-1}(n-1), \pi^{-1}(2)<\pi^{-1}(0)$ and $\pi$ is an arc permutation, letting $\pi(0):=i$ the following holds: $0=\pi^{-1}(i)<\pi^{-1}(i-1) \cdots<$ $\pi^{-1}(2)<\pi^{-1}(0)$ and $0=\pi^{-1}(i)<\pi^{-1}(i+1)<\cdots<\pi^{-1}(n-3)<\pi^{-1}(n-1)$.

It follows that for every $\pi \in U_{n}$, such that $\bar{\pi}$ is a chamber in a gallery from the identity chamber to its negative that start by flipping [0,2], there is no $(i, j)<(k, l)$ in point-wise coordinate order, with $i+1<j$ and $k+1<l$, such that $\pi^{-1}(i)<\pi^{-1}(j)$ but $\pi^{-1}(k)>\pi^{-1}(l)$. One concludes that there is no $(i, j)<(k, l)$ in point-wise coordinate order, with $i+1<j$ and $k+1<l$, such that $[i, j]$ is flipped after $[k, l]$.

It remains to prove the opposite direction, namely, to show that every linear extension of the coordinate-wise order appears as a geodesic. To prove this, first, notice that the lexicographic order does appear. Then observe that if $i<j<k<l$ and $[i, l]$ and $[j, k]$ are consequent flipped diagonals in the geodesic then it is possible to switch their order in the geodesic. This completes the proof for geodesics from $T_{0}$ to $T_{0}^{R}$, which start by flipping $[0,2]$.

Finally, to prove that geodesics from $T_{0}$ to $T_{0}^{R}$, which start by flipping $[0, n-2]$, are characterized by linear extensions with respect to the order $0 \equiv n<n-1<n-2<\cdots<$ 1 , observe that these geodesics may be obtained from geodesics that start by flipping $[0,2]$ via the reflection which maps every $0 \leqslant i \leqslant n-1$ to $n-i$.

\subsection{Skew Shifted Young Lattice}

Definition 9.2 For a positive integer $n$ let $\Lambda(n)$ be the set of all partitions with largest part $\leqslant n$ and with all parts distinct, except possibly the first two parts when they are equal to n. Namely,

$$
\begin{aligned}
\Lambda(n):=\left\{\lambda=\left(\lambda_{1}, \ldots, \lambda_{k}\right)::\right. & k \geqslant 0, n \geqslant \lambda_{1} \geqslant \lambda_{2}>\lambda_{3}>\cdots>\lambda_{k}>0 \text { and } \\
& {\left.\left[\text { either } \lambda_{1}>\lambda_{2} \text { or } \lambda_{1}=\lambda_{2}=n\right]\right\} . }
\end{aligned}
$$

Let $(\Lambda(n), \subseteq)$ the poset of partitions in $\Lambda(n)$ ordered by inclusion of the corresponding Young diagrams. 


\section{Example 9.3}

$$
\begin{aligned}
& \Lambda(3)=\{(3,3,2,1),(3,3,2),(3,3,1),(3,3), \\
& (3,2,1),(3,2),(3,1),(3),(2,1),(2),(1),() \quad\} \text {. }
\end{aligned}
$$

Consider the standard tableaux of truncated shifted staircase shape $(n-1, n-1, n-$ $2, n-3, \ldots, 2,1)$. Denote this set by $Y(n)$.

Example 9.4 The truncated shifted staircase shape $(3,3,2,1)$ is drawn in the following way:

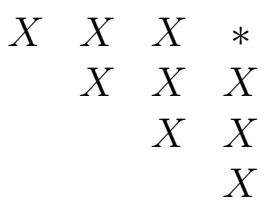

There are four standard tableaux of this shape

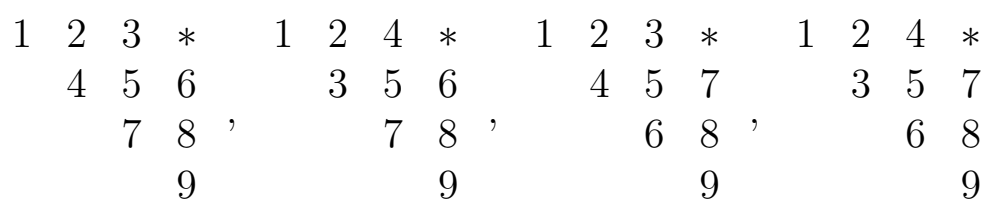

Observation 9.5 1. The maximal chains in $(\Lambda(n), \subseteq)$ are parameterized by the set of standard tableaux of truncated shifted staircase shape $(n-1, n-1, n-2, \ldots, 1)$.

2. The linear extensions of the coordinate-wise order on the set

$$
\{(i, j): 0 \leqslant i+1<j \leqslant n\} \backslash\{(0, n)\}
$$

are parameterized by the set of standard tableaux of truncated shifted staircase shape $(n-1, n-1, n-2, \ldots, 1)$.

With any standard tableau of truncated shifted staircase shape $T$ associate two words of size $\left(\begin{array}{l}n \\ 2\end{array}\right)-1, r(T)$ and $c(T)$, where $r(T)_{i}\left(c(T)_{i}\right),\left(1 \leqslant i \leqslant\left(\begin{array}{l}n \\ 2\end{array}\right)-1\right)$, is the row (respectively, column) where entry $i$ is located.

Example 9.6 Let $P, Q$ be the first two tableaux in Example 9.4. Then $r(P)=(1,1,1,2,2,2,3,3,4), c(P)=(1,2,3,2,3,4,3,4,4), r(Q)=(1,1,2,1,2,2,3,3,4)$ and $c(Q)=(1,2,2,3,3,4,3,4,4)$, 


\subsection{Geodesics and Tableaux}

Denote the set of geodesics from $T_{0} \in C T F T(n)$ to $T_{0}^{R}$ starting by flipping [0,2] by $D\left(T_{0}\right)^{+}$.

Proposition 9.7 1. There is a bijection from the set of geodesics $D\left(T_{0}\right)^{+}$to $Y(n-3)$ (the set of standard tableaux on truncated shifted staircase partition $(n-3, n-3, n-$ $4, \ldots, 1)$ )

$$
\phi: D\left(T_{0}\right)^{+} \rightarrow Y(n-3) .
$$

2. For every geodesic $u \in D\left(T_{0}\right)^{+}$, the diagonal flipped at the $i$-th step is

$$
\left[r(\phi(u))_{i}-1, c(\phi(u))_{i}+1\right]
$$

Example 9.8 The bijection $\phi$ maps the tableau

$$
\begin{array}{rrrr}
1 & 2 & 4 & * \\
& 3 & 5 & 6 \\
& & 7 & 8 \\
& & & 9
\end{array}
$$

to the series of diagonals: $[0,2],[0,3],[1,3],[0,4],[1,4],[1,5],[2,4],[2,5],[3,5]$.

Proof. Combining Theorem 9.1 with Observation 9.5(2).

Let $d_{n}$ denote the number of geodesics from the canonical star triangulation $T_{0}$ of an $n$-gon to its reverse $T_{0}^{R}$. By Proposition $9.7, d_{n} / 2$ is equal to the number of standard tableaux of truncated shifted staircase shape $(n-3, n-3,, n-4, \ldots, 1)$. Partial results regarding $d_{n}$ were stated in an early version of this preprint. Subsequently, an explicit multiplicative formula was proved by Greta Panova [9] and Ronald C. King and the authors [2].

Theorem 9.9 The number of geodesics from the canonical star triangulation $T_{0}$ of a convex $n$-gon to its reverse $T_{0}^{R}$ is

$$
d_{n}=g^{[n-6]} \cdot\left(\begin{array}{c}
N \\
4 n-15
\end{array}\right) \cdot \frac{8(2 n-9)}{n-3}=\frac{N ! \cdot 8(2 n-9)}{(4 n-15) ! \cdot(n-3)} \cdot \prod_{i=0}^{n-7} \frac{i !}{(2 i+1) !}
$$

where $g^{[n-6]}:=g^{(n-6, n-7, \ldots, 1)}$ is the number of standard Young tableaux of shifted staircase shape $(n-6, n-7, \ldots, 1)$ and $N:=n(n-3) / 2$. 


\section{References}

[1] R. M. Adin, M. Firer and Y. Roichman, Triangle Free Triangulations, Adv. Appl. Math. 45 (2010), 77-95.

[2] R. M. Adin, R. C. King and Y. Roichman, Enumeration of standard Young tableaux of certain truncated shapes, Electron. J. Combin. 18(2) (2011), The Zeilberger Festschrift volume, Paper 20, 14 pp.

[3] A. Björner and F. Brenti, Combinatorics of Coxeter groups. Graduate Texts in Mathematics, 231. Springer, New York, 2005.

[4] J. H. Conway and H. S. M. Coxeter, Triangulated polygons and frieze patterns, Math. Gaz 57 (1973), 87-94 and 175-186.

[5] P. Dehornoy, Dual presentation of Thompson's group F and flip distance between triangulations, Lecture notes (CIRM, June 2008).

[6] P. Dehornoy, The rotation distance between binary trees, Adv. Math. 223 (2010), 1316-1355.

[7] S. Elizalde and Y. Roichman, Arc permutations, preprint, 2011.

[8] P. Orlik and H. Terao, Arrangements of hyperplanes, Springer-Verlag, Berlin, 1992.

[9] G. Panova, Truncated tableaux and plane partitions, preprint 2010, arXiv:1011.0795.

[10] B. E. Sagan, Proper partitions of a polygon and $k$-Catalan numbers, Ars Combin. 88 (2008), 109-124.

[11] D. D. Sleator, R. E. Tarjan and W. P. Thurston, Rotation distance, triangulations, and hyperbolic geometry, J. Amer. Math. Soc. 1 (1988), 647-681.

[12] R. P. Stanley, personal communication. 